\title{
Reseña del libro: Derechos Económicos, Sociales y Culturales en colectivos vulnerados. Experiencias en La Pampa. Autoras: Cecilia Bertolé, Lucía Carolina Colombato y Gina Paola Rodríguez (Editoras). 2020. La Pampa. EdUNLPam
}

Book review: Economic, Social and Cultural Rights in violated groups. Experiences in La Pampa. Autbors: Cecilia Bertolé, Lucía Carolina Colombato and Gina Paola Rodriguez (Editors). 2020. La Pampa. EdUNLPam

Ivana Romina Barneix ${ }^{1}$ Universidad Nacional de La Pampa - Argentina

Revista Derechos en Acción ISSN 2525-1678/ e-ISSN 2525-1686 Año 5/No 17 Primavera 2020 (21 septiembre a 20 diciembre), 779-786 DOI: https://doi.org/10.24215/25251678e477

Recibido: 01/09/2020

Aprobado: 15/09/2020

El libro "Derechos Económicos, Sociales y Culturales en colectivos vulnerados. Experiencias en La Pampa" es el resultado de las actividades de investigación del Grupo de Trabajo

\footnotetext{
Abogada (UNLPam). Especialización en Derechos Humanos (en curso- FCEyJ-UNLPam). Diplomada en Derecho Constitucional y Derechos Humanos (Red Justicia). Diplomada en Ciencias Políticas con orientación en Políticas Públicas y Desarrollo (FILDSS). Diplomada en Genocidios y Violencias de Estado (en curso-UBA). Asistente de investigación en proyecto "Derechos Humanos, Sistemas Internacionales de Protección y Políticas Públicas. Retos desde La Pampa" (CICJ-UNLPam). Secretaria del Observatorio de Derechos Humanos (FCEyJUNLPam) Asistente del Programa Académico Institucional de Derechos Humanos (UNLPam). Email: ivanabarneix@hotmail.com (ORCID: https://orcid.org/ 0000-0003-2135-5081).
} 
de Investigación "Derechos Humanos, Sistemas Internacionales de Protección y Políticas Públicas locales" de la Facultad de Ciencias Económicas y Jurídicas de la Universidad Nacional de La Pampa.

Reseño el libro teniendo en cuenta las siguientes apreciaciones. En primer lugar, conozco a las compiladoras y su compromiso por la educación e investigación en Derechos Humanos, lo que se ve reflejado en esta obra. En segundo lugar, me he incorporado recientemente al Grupo de Trabajo de Investigación "Derechos Humanos, Sistemas Internacionales de Protección y Políticas Públicas", por lo que este libro significa ejemplo y punto de partida para continuar investigando la adaptación legislativa y operacionalización de derechos humanos en clave regional.

El libro está organizado en una introducción general y seis capítulos a modo de artículos independientes- cada uno con introducción, desarrollo, conclusiones y referencias bibliográficas propias-, pero que denotan una ilación conceptual y eje centralizado en los Derechos Económicos, Sociales, Culturales y Ambientales (DESCA).

De la introducción se advierte la perspectiva crítica desde la cual se abordan las investigaciones del Grupo de Trabajo. En la misma se anticipa que la obra abordará, en sus distintos capítulos, cuestiones atinentes al reconocimiento normativo de los DESCA en Argentina y en La Pampa, evidenciando contrastes y tensiones que dificultan su garantía y protección efectivas.

El capítulo 1 se titula "Lecturas jurisprudenciales evolutivas sobre la protección de los DESCA, a través del artículo 26 de la Convención Americana de Derechos Humanos", y por ser el más extenso de la obra merece un mayor detalle.

Mediante su introducción y subtítulos, Esteban Torroba realiza un breve recorrido histórico del surgimiento de instrumentos internacionales y señala críticamente la separación categórica de los derechos humanos, producto de la visión occidental, que privilegió a los Derechos Civiles y Políticos por sobre los Económicos, Sociales, Culturales y Ambientales. 
El capítulo referencia los instrumentos del Sistema Interamericano de Protección de Derechos Humanos sobre Derechos Económicos, Sociales, Culturales y Ambientales, desde la Carta de la Organización de los Estados Americanos (1948) hasta la Declaración Americana sobre los Derechos de los Pueblos Indígenas (2016). De la misma manera, realiza un recuento histórico desde la Conferencia Interamericana sobre Problemas de la Guerra y de la Paz (1945), hasta los debates generados en la Conferencia Especializada Interamericana sobre Derechos Humanos (1969), en torno a los precedentes del artículo 26 de la Convención Americana de Derechos Humanos $(\mathrm{CADH})$ y la incidencia del principio de progresividad en la justiciabilidad directa de los DESCA.

En esa línea, el autor del capítulo investiga y describe la exigüidad de jurisprudencia de la Corte IDH en casos de vulneración de DESCA, detallando las formas de protección indirecta adoptadas por el Alto Tribunal Interamericano a través de normas del texto convencional sobre Derechos Civiles y Políticos y no el artículo 26 de la Convención Americana de Derechos Humanos.

Sobre el final del capítulo Torroba analiza, mediante método cualitativo y técnicas de análisis documental, los antecedentes jurisprudenciales de la Corte IDH sobre DESCA que han sido útiles para alimentar una lectura evolutiva de su protección, entre finales de los años 90 -cuando el Tribunal empieza a dar respuesta concreta a los primeros planteos de vulneración de DESCA- y la actualidad.

El autor concluye que la resolución de los casos Lagos del Campo vs. Perú y Trabajadores Cesados de Petroperú y otros vs. Perú, a través de las sentencias del 31 de agosto y 23 de noviembre de 2017, respectivamente, abrieron un nuevo panorama a partir de la declaración de incumplimiento del artículo 26 de la Convención Americana de Derechos Humanos como fuente normativa directa de DESCA. Postula, luego de un vasto análisis, que las lecturas jurisprudenciales evolutivas deberían asentarse de forma inmediata en el ámbito interno de los Estados, a fin de que contribuyan a transformar realidades sociales y políticas concretas. 
En el capítulo 2, denominado "Los caminos de la concreción de derechos culturales en La Pampa. La declaratoria del Ex Centro Clandestino de Tortura y Detención Seccional I de Santa Rosa como patrimonio cultural", Lucía Colombato continúa con el análisis crítico de los DESCA, en particular de los Derechos Culturales como "declaración de principios desprovistos de garantías que los hagan efectivos" (p. 123).

La autora realiza aportes para definir los derechos culturales y analiza las dificultades generadas en torno a que el bien jurídico protegido es colectivo y no individual. En ese sentido, se explaya sobre las distintas definiciones doctrinarias de la cultura y asume la posición conceptual de "procesos culturales", concluyendo que "la cultura es el espacio en el que se construye la hegemonía, pero también la zona en que nacen los procesos contrahegemónicos" (p. 128), donde individuos y grupos sociales se empoderan, y construyen y transmiten transgeneracionalmente referencias simbólicas.

En el capítulo, Colombato analiza los desarrollos recientes en el campo de los derechos culturales en referencia al derecho a la memoria y los insumos generados a partir de la Convención sobre la Diversidad de las Expresiones Culturales (2005). Referencia normativa nacional y provincial que destaca el patrimonio cultural como derecho humano, y describe cómo los lugares de memoria, vinculados a la última dictadura militar argentina, ganaron presencia en espacios públicos para contribuir al desagravio de violaciones a derechos humanos y generar conciencia y garantía de no repetición.

En ese marco, desde un análisis regional, Colombato describe la incorporación al Registro Provincial de Patrimonio Cultural de dos sitios que tienen vinculación con la memoria de la última dictadura cívica militar: la Escuela $N^{\circ} 286$ de Paso de los Algarrobos (Dictamen n ${ }^{\circ} 22$ ) 14 y el edificio de la Ex Escuela Hogar (Dictamen $N^{\circ}$ 14) a la vez que estudia y desarrolla particularmente la experiencia de la incorporación del Ex Centro Clandestino de Tortura y Detención Seccional I de Santa Rosa al Registro Provincial de Patrimonio Cultural (Ley No 2083). 
Al final del capítulo la autora remarca la relevancia de los derechos culturales para la realización plena de los derechos humanos, y concluye que los desafíos futuros buscan llevar a los derechos culturales a otro nivel de desarrollo, fortaleciendo "su papel en la lucha frente a las desigualdades sociales culturalmente construidas" (p. 136).

En el capítulo 3, denominado "Derechos Sexuales, Reproductivos y No Reproductivos en La Pampa: el caso de la Guía ILE", la autora Gina Paola Rodríguez problematiza el proceso de incorporación de los Derechos Sexuales, Reproductivos y No Reproductivos en La Pampa, como parte del derecho a la salud.

La autora comienza con la definición, alcance y contenido de estos derechos, para lo que retoma el esquema de Brown que los define como un concepto que incluye " 1 . La seguridad en la reproducción y los eventos relacionados con ella, 2. Anticoncepción y aborto, o aspectos de la opción no reproductiva, y 3. El libre ejercicio de la sexualidad" (p. 140). A su vez, incorpora en su análisis el proceso histórico en el que converge la demanda por el aborto legal y métodos anticonceptivos, y la demanda por el ejercicio de otras sexualidades.

En esa línea, Rodríguez procede a la reconstrucción, análisis y descripción de los hitos legislativos en la materia en La Pampa, como el Programa Provincial de Procreación Responsable creado mediante ley $\mathrm{N}^{\mathrm{o}} 1363$ (1991) y la inclusión de prácticas quirúrgicas de infertilización en la regulación del ejercicio de actividades de salud, mediante Ley N 2079 (2003), que fueron atravesados por conflictos con sectores denominados conservadores y religiosos. La autora concluye que en una primera etapa los derechos sexuales y (no) reproductivos en La Pampa se configuraron en torno a "la institución matrimonial y familiar y el control del cuerpo de las mujeres" (p. 147).

En el mismo sentido, el capítulo presenta un barrido histórico sobre el camino sinuoso y conflictivo del aborto no punible en La Pampa, iniciado con el proyecto de ley No 2394 de 2007 y el veto del Poder Ejecutivo producto de pujas sociales y jurisprudenciales que giraron en torno a dicho proyecto la ley. 
Sobre el final del capítulo, Rodríguez analiza el impacto del fallo FAL (2012) en la regulación del aborto no punible en La Pampa, y detalla las insuficiencias y restricciones del Protocolo de Aborto no Punible (Resolución No 656/2012), modificada y enmendada la Resolución No 1789/18, que creó la Guía Provincial de Atención Integral de las Personas con Derecho a la Interrupción Legal del Embarazo. Luego de realizar un análisis de los movimientos en la política argentina que permitieron la Guía, se enfoca en los desafíos de su implementación.

La autora concluye el capítulo con un mensaje esperanzador en la materia, al determinar que, si bien persisten barreras formales e informales que ameritan una mayor acción estatal en materia de consecuencias administrativas, civiles y penales frente a casos de responsabilidad por obstrucción y/o falta de provisión de servicios, existen argumentos para concluir que "La Pampa se halla más cerca de garantizar los derechos sexuales y (no) reproductivos que otras provincias de la Argentina"(p. 160).

Por su parte, en el capítulo 4, denominado "Debates en relación con la puesta en funcionamiento del Sistema de Promoción y Protección Integral de derechos de niños, niñas y adolescentes en la provincia de La Pampa", la autora Cecilia Bertolé analiza el tenso proceso de adaptación legal, a nivel nacional y en la provincia de La Pampa, del Sistema de Promoción y Protección Integral de derechos de niños, niñas y adolescentes (NNyA).

Mediante técnicas de investigación cualitativa, Bertolé describe y destaca los avances normativos en materia de niñez en la provincia, como la ley No 2703 de 2013 que ha adherido al nuevo paradigma integral instaurado por la Convención de los Derechos del Niño, el "Protocolo de intervención en situaciones de abuso sexual contra niños, niñas y adolescentes" del año 2017 y la "Guía de procedimiento del Sistema de Protección, Promoción y Restitución de derechos de la niñez y adolescencia de la Provincia de la Pampa" de 2018.

En contraste, y como resultado de entrevistas a integrantes claves del Sistema en La Pampa, diagnostica y sintetiza en siete 
puntos las dificultades en términos de exigibilidad de esos derechos, como ser la falta de capacitación de los actores del sistema y obstaculizaciones para implementación de los principios de descentralización, desjudicialización y corresponsabilidad.

El capítulo evidencia el argumento central del libro, en cuanto permite concluir que el reconocimiento normativo de derechos es sólo el primer paso para su garantía y goce efectivo; e invita a reflexionar sobre el camino que aún falta recorrer en torno a la construcción del sistema de promoción y protección integral en la provincia de La Pampa que coloque a niños, niñas y adolescentes en el centro del proceso.

En el capítulo 5, titulado "Acceso a la justicia de personas con discapacidad: un derecho humano fundamental y una garantía para el ejercicio de todos sus derechos", la autora Eliana Mariel Ferrero describe los alcances del Acceso a la Justicia de las personas con discapacidad y lo aborda desde un doble carácter.

En primer lugar, como derecho humano en sí mismo, detalla la regulación normativa en el derecho internacional, y posteriormente, adentrándose en la discapacidad como una cuestión de derechos humanos, analiza normativa nacional e internacional y estándares jurisprudenciales y doctrinarios que determinan el acceso a la justicia como una garantía para el ejercicio de todos los derechos de las personas con discapacidad.

Desde ese doble enfoque, Ferrer evidencia las barreras arquitectónicas, comunicacionales y actitudinales que dificultan el acceso a la justicia de las personas con discapacidad y, con ello, la vigencia de otros derechos. En ese sentido, concluye con el detalle de instrumentos internacionales, como el "Protocolo para el Acceso a la Justicia de las Personas con Discapacidad: Propuestas para un trato adecuado" del EUROSOCIAL y las "Reglas de Brasilia", que recomiendan la implementación de ajustes y buenas prácticas para la remoción de dichas barreras.

El libro culmina con el capítulo 6, que se titula "La conceptualización del sujeto de derechos en la Convención Interamericana sobre la protección de los Derechos Humanos de las 
Personas Mayores. ¿Protección desde una perspectiva regional?”. En él, el autor Maximiliano Corroinca problematiza las definiciones sobre Personas Mayores, enmarcadas en un contexto global que se sintetiza como de menoscabo de derechos económicos sociales y culturales del colectivo elegido y escaso nivel de respuestas público-estatales en cuanto a políticas del cuidado.

Corroinca describe la visión de la vejez en los instrumentos normativos y estándares internacionales, analiza la perspectiva regional de la Convención, aprobada por ley 27360 del Congreso de la Nación, y adhesión mediante ley 3029 de la Provincia de La Pampa, y concluye en el carácter tuitivo de la Convención como preventivo, en función de las particularidades del colectivo estudiado, e invita a partir de "una disputa de sentido crítica en la búsqueda de definiciones tuitivas para la población, que garanticen y eventualmente promuevan el bienestar de sus derechos y prerrogativas" (p. 222)

En conclusión, el libro analiza la tensión entre reconocimiento normativo y goce efectivo de Derechos Económicos, Sociales, Culturales y Ambientales, debido a las particularidades que reviste este grupo de derechos, tal como analizaron Torroba y Colombato. A su vez, las y los autoras/es Rodriguez, Bertolé, Ferrer y Corroinca analizan avances y retrocesos, pasados y actuales, en materia de derechos de derechos de niñas, niños $\mathrm{y}$ adolescentes, mujeres y cuerpos gestantes, personas con discapacidad y adultos mayores, evidenciando la dificultad de acceso al goce de derechos de esos colectivos vulnerados.

Debe destacarse que el libro muestra el compromiso con la defensa y promoción de los derechos humanos por parte del Grupo de Trabajo y, al socializar los resultados de la investigación con esta obra, impacta de manera positiva en actuales y futuros investigadores de la región pampeana. 